\title{
Cox Regression With Covariates Missing Not At Random
}

\author{
Victoria J. Cook ${ }^{1}$, X. Joan $\mathrm{Hu}^{2}$ and Tim B. Swartz ${ }^{2}$ \\ ${ }^{1} B C$ Centre for Disease Control and University of British Columbia \\ ${ }^{2}$ Simon Fraser University
}

\begin{abstract}
This paper considers estimation under the Cox proportional hazards model with rightcensored event times in the presence of covariates missing not at random (MNAR). We propose an approach derived from likelihood estimation utilizing supplementary information. We show that available additional information not only helps to account appropriately for the missing covariates but also leads to estimation procedures which are natural and easy to implement. A medical example is used throughout the paper to motivate the problem and to illustrate the proposed methodology.

Key words: event time; missing data; semiparametric estimation; sensitivity analysis; supplementary information.
\end{abstract}

\section{Introduction}

In many practical studies, the observation of an event time is subject to right-censoring and measurements on certain components of the covariates are not available on all study subjects. It is often of interest to estimate the parameters in the Cox proportional hazards model with such missing data. To avoid the computational burden induced by the unspecified baseline function in the model, many attempts to tackle the problem employ variations 
of the partial likelihood estimating functions based on the right-censored event times with complete covariate observations. See, for example, Lin and Ying (1993), Zhou and Pepe (1995), Paik and Tsai (1997) and Qi, Wang and Prentice (2005). Notable exceptions include Chen and Little (1999) and Herring and Ibrahim (2001), which specify parametrically the distribution of the covariate and propose likelihood based estimation procedures. Although there has been some excellent work on data missing not at random (MNAR) (e.g., Scharfstein, Rotnitzky and Robins, 1999), most published papers in this area assume that the missing covariates are either missing completely at random (MCAR) or missing at random (MAR).

Tuberculosis (TB) is an infectious disease spread by the airborne route. There are many risk factors that increase a person's chance of being infected or going on to develop active disease. HIV infection is the single greatest risk factor associated with TB. Motivated by a research question at the Division of TB Control, Centre for Disease Control of British Columbia (BCCDC), we consider Cox regression with covariates missing not at random (MNAR). An investigation was conducted to evaluate the association of the time to TB and latent TB infection (LTBI) with a list of potential risk factors based on subjects in the Greater Vancouver area who had contact with active infectious TB patients (i.e., source cases); see Cook, et al (2005). A total of 7,921 people were identified as contacts from the TB registry and were taken as study subjects. The time to TB development of each TB contact was collected up to October 2003. This, together with the staggered study entries, resulted in the observation of the event times (i.e., times to TB since the contacts) subject to a noninformative right-censoring. Excluding HIV status, information on all of the potential risk factors was available from $97.9 \%$ of the study subjects $(7,754$ out of 7,921$)$. With 
respect to the HIV status covariate, only $2.2 \%$ of the study subjects (176 out of 7,921 ) had HIV test results documented in the TB registry. This lack of complete data resulted from the data collection process due to a TB policy, which is to test all active TB cases for HIV co-infection but not all TB contacts. However, nurses interviewed the TB contacts and requested HIV testing in those contacts with risk factors of HIV infection. Thus the missingness of a subject's HIV status could be closely associated with the presented lack of risks for HIV infection. In other words, the missingness of the covariate likely depends on the covariate measurement. This type of missingness is also referred to as nonmissing at random (NMAR) in the literature (c.f., Tsiatis, 2006). The well-established inverse probability weighted complete-case estimator (Robins, Rotnitzky and Zhao, 1994) and its extensions, augmented inverse probability weighted complete-case estimators (c.f., Bang and Robins, 2005), are not directly applicable in handling the current missing data, and neither is the commonly-used multiple imputation approach (c.f., Little and Rubin, 2002).

Attempts were made to adapt the conventional approach under the Cox proportional hazards model for right-censored survival times when the covariates are fully observed. They included focusing on the data of the complete cases, having the indicator of missingness as an additional covariate in the analysis, and imputing all the missing HIV entries as negative. None of these approaches appeared plausible or produced satisfactory analysis outcomes as they would lead to either an obviously over or under estimation of the population HIV status in the TB contacts. As pointed out in Tsiatis (2006), analysis of data with MNAR missingness is in general problematic, since the missingness may depend on unobserved data and adjustments for the missing data cannot be verified with the available data. These considerations led us to explore the problem utilizing additional information. 
In many practical situations, there is supplementary information on the covariate components whose values associated with a portion of the study subjects are missing. This type of information is readily available in some studies. For example, the TB contact study team collected the prevalence information of HIV infection in different cohorts, such as various groups of TB contacts and the general population in the Great Vancouver area (or, in British Columbia). Other studies conducted a follow-up survey on the individuals who had missing covariate measurements; see Suzuki (1985) for an example of this with car warranty data. Hu and Lawless $(1996,1997)$ present parametric estimation approaches based on right-censored failure times with several types of supplementary information on the missing censoring times. In the present work, we extend their methods to a semiparametric setting with MNAR covariates. We assume that the time to an event of interest $T$ follows the Cox proportional hazards model, and present estimation procedures built upon the likelihood function from right-censored event times with MNAR covariates in combination with additional information on the missing covariates from another source. Procedures associated with supplementary information in different forms are developed for computing the estimators of the regression parameters and the unspecified baseline function. They are easy to follow and implement.

The rest of the paper is organized as follows. Section 2 introduces the framework of the proposed approach. Sections 3 and 4 describe procedures for estimating the regression parameters in the Cox model with the available data in combination with certain supplementary information. Section 5 reports the preliminary analyses of missing HIV status in TB contacts, and analyzes the TB contact data augmented with HIV prevalence information. It also presents sensitivity analyses conducted to verify adjustments for the missingness of 
HIV entries. Some final remarks are given in Section 6.

\section{Notation, Modelling and Assumptions}

We formulate the aforementioned problem as follows. Suppose that an event time $T$ conditional on covariates $(X, Z)$ follows the Cox proportional hazards model (Cox, 1972): its conditional hazard function is

$$
h(t \mid X, Z)=h_{0}(t) \exp \left(\alpha^{\prime} X+\beta^{\prime} Z\right), t>0
$$

Let $\left\{\left(T_{i}, X_{i}, Z_{i}\right): i=1, \ldots, n\right\}$ be i.i.d. realizations of $(T, X, Z)$, associated with a study with $n$ individuals, where $Z$ and $X$ denote the covariate components with and without entries from all the individuals, respectively. Our primary interest is in estimating the regression coefficients $\alpha$ and $\beta$ in the Cox model (1) from the study's available data:

$$
\left\{\left(U_{i}, \Delta_{i}, R_{i} X_{i}, R_{i}, Z_{i}\right): i=1, \ldots, n\right\}
$$

where $U_{i}=T_{i} \wedge C_{i}$ is the minimum of $T_{i}$ and $C_{i}$ with $C_{i}$ the censoring time of subject $i$, the variable $\Delta_{i}$ indicates $T_{i} \leq C_{i}$ or not, and $R_{i}$ is the missing indicator for whether $X_{i}$ is observed. We focus on the situations where the MAR (missing at random) assumption, $P(R \mid U, \Delta, X, Z)=P(R \mid U, \Delta, Z)$, does not necessarily hold, such as the TB contact study described in Section 1.

We make the following two assumptions throughout the paper, which are plausible in many practical situations:

A1. The censoring is conditionally noninformative: $T \perp C \mid(X, Z)$. 
A2. Conditional on $(X, Z),(U, \Delta)$ is independent of whether $X$ is observed or not: $P(U, \Delta \mid X, Z, R)=$ $P(U, \Delta \mid X, Z)$

Using the generic notation for densities, the information contributed by the available data of an individual under assumptions $\mathrm{A} 1$ and $\mathrm{A} 2$ is

$$
[U, \Delta \mid X, Z][X \mid R=1, Z][R=1, Z]
$$

when $R=1$;

$$
\int[U, \Delta \mid X, Z] d[X \mid R=0, Z][R=0, Z]
$$

when $R=0$. Further, denote the distribution function of $X$ given $R$ and $Z=z$ by $G_{R}(\cdot \mid z)$, i.e., $G_{R}(x \mid z)=P(X \leq x \mid R, Z=z)$. The distribution of $X$ given $Z=z$ is then $G(x \mid z)=p(z) G_{1}(x \mid z)+(1-p(z)) G_{0}(x \mid z)$, where $p(z)=P(R=1 \mid Z=z)$. Here $G_{1}(\cdot \mid z)$ and $G_{0}(\cdot \mid z)$ are the conditional distributions of the observed $X$ and unobserved $X$ given $Z=z$, respectively. The likelihood function based on the available data (2) is proportional to

$$
\begin{aligned}
& L_{F}\left(\alpha, \beta, h_{0}, G_{0}, G_{1}, p\right)=\prod_{i=1}^{n}\left\{h\left(T_{i} \mid X_{i}, Z_{i}\right)^{\Delta_{i}} S\left(U_{i} \mid X_{i}, Z_{i}\right) g_{1}\left(X_{i} \mid Z_{i}\right) p\left(Z_{i}\right)\right\}^{R_{i}} \\
\times & \left\{\int h\left(T_{i} \mid x, Z_{i}\right)^{\Delta_{i}} S\left(U_{i} \mid x, Z_{i}\right) d G_{0}\left(x \mid Z_{i}\right)\left(1-p\left(Z_{i}\right)\right)\right\}^{1-R_{i}},
\end{aligned}
$$

where the conditional survivor function $S(t \mid x, z)=\exp \left\{-H_{0}(t) e^{\alpha^{\prime} x+\beta^{\prime}} z\right\}$ with the cumulative baseline function $H_{0}(t)=\int_{0}^{t} h_{0}(u) d u$ for $t \geq 0$, and $g_{1}(\cdot \mid z)$ is the density function or probability mass function associated with $G_{1}(\cdot \mid z)$.

In what follows, we explore the estimation of $\alpha$ and $\beta$ as well as $H_{0}(\cdot)$ based on the likelihood function (3) in situations with certain supplementary information on the unobserved $X_{i}$. We start by assuming that $G_{0}(\cdot \mid z)$, the conditional distribution of the unobserved 
covariates given $Z=z$, is known. This is the simplest case which serves as an introduction to more practical scenarios. We use the lower case of a letter to represent a realization of the random variable (vector) denoted by the upper case of the corresponding letter in formulas throughout the paper; for example, $r_{i}$ is a realization of $R_{i}$ in (2).

\section{Estimation with Known $G_{0}(\cdot \mid z)$}

In this case, the likelihood function (3) reduces to

$L\left(\alpha, \beta, h_{0}(\cdot)\right)=\prod_{i=1}^{n}\left\{h\left(T_{i} \mid X_{i}, Z_{i}\right)^{\Delta_{i}} S\left(U_{i} \mid X_{i}, Z_{i}\right)\right\}^{R_{i}}\left\{\int h\left(T_{i} \mid x, Z_{i}\right)^{\Delta_{i}} S\left(U_{i} \mid x, Z_{i}\right) d G_{0}\left(x \mid Z_{i}\right)\right\}^{1-R_{i}}$.

With a given realization of (2) as the available data, the likelihood function (4) involves only finite dimensional parameters $\alpha, \beta$ and $\theta=\left(h_{0}\left(u_{1}^{*}\right), \ldots, h_{0}\left(u_{J}^{*}\right)\right)^{\prime}$, where $u_{j}^{*}, j=1, \ldots, J$ are the distinct values of the realizations of $U_{i}, i=1, \ldots, n$. Here $J \leq n$ and the equal sign holds when there are no tied observation times. Following Breslow (1972), we maximize $L\left(\alpha, \beta, h_{0}(\cdot)\right)$ in $(4)$ viewing $h_{0}(u) \neq 0$ only when $u=u_{j}^{*}, j=1, \ldots, J$, and thus consider the likelihood estimating equations,

$$
\partial \log L\left(\alpha, \beta, h_{0}(\cdot)\right) / \partial \theta=0, \quad \partial \log L\left(\alpha, \beta, h_{0}(\cdot)\right) / \partial(\alpha, \beta)^{\prime}=0
$$

Using the counting process notation, let $Y_{i}(t)=I\left(U_{i} \geq t\right)$ and $N_{i}(t)=I\left(T_{i} \leq t\right)$ denote the at risk indicator and the survival indicator of subject $i$ at time $t$, respectively. The first set of equations in (5) can be written as

$$
H_{0}(t ; \alpha, \beta)=\sum_{i=1}^{n} \int_{0}^{t} \frac{Y_{i}(s) d N_{i}(s)}{\sum_{j=1}^{n} Y_{j}(s)\left[R_{j} e^{\alpha X_{j}}+\left(1-R_{j}\right) e^{\widehat{\alpha X_{j}}}\right] e^{\beta Z_{j}}}, \quad t>0
$$


where

$$
e^{\widehat{\alpha X}}=\mathrm{E}\left[e^{\alpha X} \mid U_{i}, \Delta_{i}, Z_{i}, R_{i}=0\right]=\int e^{\alpha x} d G\left(x \mid U_{i}, \Delta_{i}, R_{i}=0, Z_{i} ; \alpha, \beta, h_{0}(\cdot)\right)
$$

with the distribution of $X$ conditional on the observed data $\left(U_{i}, \Delta_{i}, R_{i}=0, Z_{i}\right)$ given as

$$
d G\left(x \mid U_{i}, \Delta_{i}, R_{i}=0, Z_{i} ; \alpha, \beta, h_{0}(\cdot)\right)=\frac{e^{\Delta_{i} \alpha x} \exp \left\{-H_{0}\left(U_{i}\right) e^{\alpha x+\beta Z_{i}}\right\} d G_{0}\left(x \mid Z_{i}\right)}{\int e^{\Delta_{i} \alpha x} \exp \left\{-H_{0}\left(U_{i}\right) e^{\alpha x+\beta Z_{i}}\right\} d G_{0}\left(x \mid Z_{i}\right)}
$$

The right-hand side of (6) with fixed $\alpha$ and $\beta$ is the commonly-used Breslow estimator for the cumulative baseline function plugged in with $\widehat{e}^{\widehat{\alpha X}_{i}}$ given in (7), an "estimator" of $e^{\alpha X_{i}}$ for subject $i$ with $X_{i}$ unobserved. It cannot be used directly as an estimator of the baseline $H_{0}(\cdot)$, for it involves the unknown $H_{0}(\cdot)$ through $\widehat{e}^{\alpha X_{i}}$. The equation $(6)$, however, motivates a natural estimation procedure, a self-consistent procedure, for $H_{0}(\cdot)$ with fixed $\alpha$ and $\beta$.

Further, combining (6) with the second set in (5) yields the following estimating equations:

$$
\sum_{i=1}^{n} \int_{0}^{\infty} Y_{i}(t)\left[\left\{R_{i} X_{i}+\left(1-R_{i}\right) \widehat{X}_{i}\right\}-\frac{S_{\alpha}^{(1)}(t ; \alpha, \beta)}{S^{(0)}(t ; \alpha, \beta)}\right] d N_{i}(t)=0
$$

and

$$
\sum_{i=1}^{n} \int_{0}^{\infty} Y_{i}(t)\left[Z_{i}-\frac{S_{\beta}^{(1)}(t ; \alpha, \beta)}{S^{(0)}(t ; \alpha, \beta)}\right] d N_{i}(t)=0
$$

where

$$
\begin{gathered}
S^{(0)}(t ; \alpha, \beta)=\frac{1}{n} \sum_{i=1}^{n} Y_{i}(t)\left[R_{i} e^{\alpha X_{i}}+\left(1-R_{i}\right) \widehat{e^{\alpha X_{i}}}\right] e^{\beta Z_{i}}, \\
S_{\alpha}^{(1)}(t ; \alpha, \beta)=\frac{1}{n} \sum_{i=1}^{n} Y_{i}(t)\left[R_{i} X_{i} e^{\alpha X_{i}}+\left(1-R_{i}\right) \widehat{X_{i} e^{\alpha} X_{i}}\right] e^{\beta Z_{i}},
\end{gathered}
$$

and

$$
\left.S_{\beta}^{(1)}(t ; \alpha, \beta)=\frac{1}{n} \sum_{i=1}^{n} Y_{i}(t)\left[R_{i} e^{\alpha X_{i}}+\left(1-R_{i}\right) \widehat{e}^{\alpha X_{i}}\right] Z_{i} e^{\beta Z_{i}}\right]
$$


with

$$
\widehat{X_{i}}=\mathrm{E}\left[X \mid U_{i}, \Delta_{i}, R_{i}=0, Z_{i}\right]=\int x d G\left(x \mid U_{i}, \Delta_{i}, R_{i}=0, Z_{i} ; \alpha, \beta, h_{0}(\cdot)\right)
$$

and

$$
\widehat{X_{i} e^{\alpha} X_{i}}=\mathrm{E}\left[X e^{\alpha X} \mid U_{i}, \Delta_{i}, R_{i}=0, Z_{i}\right]=\int x e^{\alpha x} d G\left(x \mid U_{i}, \Delta_{i}, R_{i}=0, Z_{i} ; \alpha, \beta, h_{0}(\cdot)\right)
$$

Note that (9) and (10) are the familiar partial likelihood estimating equations with the $X_{i}, e^{\alpha X_{i}}$ and $X_{i} e^{\alpha X_{i}}$ associated with the subjects without observed $X_{i}$ (i.e., with $R_{i}=0$ ) replaced by $\widehat{X}_{i}, \widehat{e^{\alpha X}}, \widehat{X_{i} e^{\alpha} X_{i}}$, respectively. The two estimating equations (9) and (10) also involve the unknown baseline and cannot be used directly to estimate $(\alpha, \beta)$. The following describes an algorithm for computing the solution to the likelihood estimating equations (5), i.e., the maximum likelihood estimator (MLE) of $\left(\alpha, \beta, h_{0}(\cdot)\right)$, utilizing jointly $(9),(10)$ and (6) with a realization of the available data (2).

Algorithm 1. With the current estimates of the unknowns, $\alpha^{(k-1)}, \beta^{(k-1)}$ and $H_{0}^{(k-1)}(\cdot)$ for $k \geq 1$,

Step 1: For each individual with $r_{i}=0$,

Step 1A: based on (8), obtain the conditional distribution

$$
G_{0 i}^{(k-1)}(x)=G\left(x \mid u_{i}, \delta_{i}, r_{i}=0, z_{i} ; \alpha^{(k-1)}, \beta^{(k-1)}, h_{0}^{(k-1)}(\cdot)\right)
$$

Step 1B: calculate the conditional expectations $\widehat{x_{i}}, \widehat{e^{\alpha x_{i}}}$, and $\widehat{x_{i} \widehat{e}^{\alpha} x_{i}}$ by plugging $G_{0 i}^{(k-1)}(x)$ in (11), (7), and (12), respectively.

Step 2: Solve the equations (9) and (10) jointly to obtain the updated estimates $\alpha^{(k)}$ and $\beta^{(k)}$. 
Step 3: Plug $\alpha^{(k)}$ and $\beta^{(k)}$ as well as $H_{0}^{(k-1)}(\cdot)$ in the right-hand side of (6) to obtain the updated baseline estimate $H_{0}^{(k)}(\cdot)$.

Repeat Steps 1-3 until the sequence $\left\{\left(\alpha^{(k)}, \beta^{(k)}, H_{0}^{(k)}(\cdot)\right): k=0, \ldots,\right\}$ converges. Denote the limit by $\left(\hat{\alpha}\left(G_{0}\right), \hat{\beta}\left(G_{0}\right), \hat{H}_{0}\left(\cdot ; G_{0}\right)\right)$.

Algorithm 1 may be viewed as an application of the EM algorithm (Dempster, Laird and Rubin, 1977). Viewing the right-censored data in the absence of missing covariates as the "full data", Step 1 above is then the E-step and Steps 2 and 3 together function as the M-step. Thus, under fairly general conditions, the algorithm converges to the MLE of the parameters in the Cox model (1). We may use the estimates of the model parameters from the complete cases by the conventional approach as the starting values $\alpha^{(0)}, \beta^{(0)}$ and $H_{0}^{(0)}$.

We need to investigate the existence of the $\operatorname{MLE}\left(\hat{\alpha}\left(G_{0}\right), \hat{\beta}\left(G_{0}\right), \hat{H}_{0}\left(\cdot ; G_{0}\right)\right)$ along the lines of Chen, Ibrahim and Shao (2009). The martingale approach that is commonlyused to derive asymptotic properties of the MLE in related situations (c.f., Andersen, et al, 1992) is not applicable here, since the processes $B_{i}(\cdot)$ in the expression $\sum_{i=1}^{n} \int_{0}^{\infty} B_{i}(t) d N_{i}(t)$ appearing in (9), for example, are not all predictable. We need to use the empirical process theory to verify the consistency, the asymptotic normality and the weak convergence of the MLE, following the arguments in, for example, Zeng and Cai (2005) and Nan, Kalbfleisch and $\mathrm{Yu}(2009)$.

Note that (9) and (10) cannot be directly used for variance estimation of $\hat{\alpha}\left(G_{0}\right)$ and $\hat{\beta}\left(G_{0}\right)$, since they involve the unspecified baseline, of which the estimator is a function of $\hat{\alpha}\left(G_{0}\right)$ and $\hat{\beta}\left(G_{0}\right)$. In principle, with a particular realization of the available data (2), we can estimate the asymptotic variances of $\hat{\alpha}\left(G_{0}\right)$ and $\hat{\beta}\left(G_{0}\right)$ using the corresponding submatrices of the inverse of the matrix $-\partial^{2} \log L\left(\alpha, \beta, h_{0}(\cdot)\right) / \partial(\alpha, \beta, \theta)^{2}$ with the unknowns 
evaluated at $\hat{\alpha}\left(G_{0}\right), \hat{\beta}\left(G_{0}\right)$ and $\hat{H}_{0}\left(\cdot ; G_{0}\right)$. The dimension of the matrix is usually very large. An alternative is to employ the bootstrap approach to achieve a variance estimate of the estimator (c.f., Efron and Tibshirani, 1993).

\section{Estimation with Partial Information on $G_{0}(\cdot \mid z)$}

We now consider a situation where there is information on the unobserved covariates but the distribution is not fully specified. We assume that there is a consistent estimator for $G_{0}(\cdot \mid z)$, denoted by $\tilde{G}_{0}(\cdot \mid z)$. For example, such an estimator can be easily obtained with a follow-up survey conducted on a randomly selected subgroup of the individuals with unobserved $X_{i}$. Then a natural estimation procedure is to implement the procedure described in Section 3 with the unknown $G_{0}(\cdot \mid z)$ replaced by the estimator $\tilde{G}_{0}(\cdot \mid z)$. This approach yields $\hat{\alpha}\left(\tilde{G}_{0}\right), \hat{\beta}\left(\tilde{G}_{0}\right)$ and $\hat{H}_{0}\left(\cdot ; \tilde{G}_{0}\right)$, which maximize the pseudo-likelihood function $L\left(\alpha, \beta, h_{0}(\cdot) ; \tilde{G}_{0}\right)$, attained by plugging $\tilde{G}_{0}(\cdot \mid z)$ in $(4)$.

Generally speaking, properties of the maximum pseudo-likelihood estimator (MPSLE) depend on how well $\tilde{G}_{0}(\cdot \mid z)$ estimates $G_{0}(\cdot \mid z)$ and how the estimator $\tilde{G}_{0}(\cdot \mid z)$ is associated with the likelihood function (4). See, for example, the discussion in Hu and Lawless (1997) about MPSLE under a parametric model in the cases with supplementary follow-up data or independent supplementary data. Preliminary simulations indicate that the MPSLE performs similarly to the MLE with known $G_{0}(\cdot \mid z)$ given in Section 3 when $\tilde{G}_{0}(\cdot \mid z)$ is close to $G_{0}(\cdot \mid z)$.

Information on the covariate $X$ over the entire population is sometimes easier to collect. With the issue of missing HIV status in TB contacts, HIV prevalence information 
in British Columbia is readily available from the literature (c.f., HIV/AIDS Epi Updates 2007, Public Health Agency of Canada). The information may be used to specify the conditional distribution of HIV status in the general population, that is, $G(\cdot \mid z)=G\left(\cdot \mid z^{(1)}\right)$ with $z^{(1)}$ a component vector of $z$. The available data (2) provide convenient estimators for $p\left(z^{(1)}\right)=P\left(R=1 \mid z^{(1)}\right)$ and $G_{1}\left(\cdot \mid z^{(1)}\right)$ when the component vector $Z^{(1)}$ is discrete. For example, denoting $n_{1}\left(z^{(1)}\right)=\sum_{i=1}^{n} R_{i} I\left(Z_{i}^{(1)}=z^{(1)}\right)$ and $n\left(z^{(1)}\right)=\sum_{i=1}^{n} I\left(Z_{i}^{(1)}=z^{(1)}\right)$, we can use $\hat{p}\left(z^{(1)}\right)=n_{1}\left(z^{(1)}\right) / n\left(z^{(1)}\right)$ and $\hat{G}_{1}\left(x \mid z^{(1)}\right)=\sum_{i: R_{i}=1} I\left(X_{i} \leq x, Z_{i}^{(1)}=z^{(1)}\right) / n_{1}\left(z^{(1)}\right)$, the proportion of subjects with observed $X_{i}$ and the empirical distribution of the observed $X_{i}$ among those with $Z_{i}^{(1)}=z^{(1)}$, respectively. Given $G(\cdot \mid z)=G\left(\cdot \mid z^{(1)}\right)$, we have

$$
\tilde{G}_{0}\left(x \mid z^{(1)}\right)=\left\{G\left(x \mid z^{(1)}\right)-\hat{p}\left(z^{(1)}\right) \hat{G}_{1}\left(x \mid z^{(1)}\right)\right\} /\left(1-\hat{p}\left(z^{(1)}\right)\right)
$$

which is a uniformly consistent estimator for $G_{0}\left(\cdot \mid z^{(1)}\right)$, provided $0<p\left(z^{(1)}\right)<1$ for $\forall z^{(1)}$.

In practice, it is often the case that $G(\cdot \mid z)=G\left(\cdot \mid z^{(1)}\right)$ is an assumption and $G\left(\cdot \mid z^{(1)}\right)$ is only roughly known. When applying the pseudo-likelihood procedure aforementioned, we suggest a sensitivity analysis.

\section{TB Contact Example}

\subsection{Preliminaries}

The initial analyses of development to TB in TB contacts at the BCCDC assumed the Cox proportional hazards model (1), and focused on the information from the 7,754 study subjects who had entries of the right-censored event times and all the covariates but the covariate of HIV status. A thorough list of risk factors likely associated with the time to TB development was identified (Canadian Tuberculosis Standards, 2007). For illustrative 
purposes, we report the analyses with a shorter list of risk factors, including only genotype cluster-status of TB source case, gender, HIV status, age at contact, and level of contact. The covariate $X$ in the general framework is the indicator of HIV infection with the TB contact study.

The initial attempt considered the Cox partial likelihood estimation approach. The study data were analyzed by (i) removing HIV status from the covariate list, (ii) focusing on the complete cases (i.e., only using the data from the 171 subjects who had confirmation of HIV test results), (iii) imputing all the missing HIV entries as negative, and (iv) introducing the indicator of HIV status missingness as an additional covariate. Table 1 presents the maximum partial likelihood estimates (MPLE) of the regression parameters and the pvalues of the associated significance tests based on the asymptotic normality of the MPLE with those data manipulations.

\section{(Table 1 is here.)}

In order to fully understand the time to development of $\mathrm{TB}$, it is necessary to keep the risk factor of HIV status in the analysis. As such, the first analysis excluding HIV as a risk factor was undesirable. The analysis of the complete cases could be meaningful with respect to the entire study population only under the MCAR assumption, which was also not plausible in this application as the observed HIV infection rate was 11.1\% (19 out of 171), higher than the HIV infection rates of the various groups of TB contacts in the literature and locally (e.g., Reichler, et al, 2003). The third analysis assumed accurate assessments by the nurses based on their short interviews with the study subjects where the missing HIV status was imputed as negative. The HIV infection rate of the subjects with the imputed data was $0.2 \%$, lower than the HIV prevalence of $0.3 \%$ in $\mathrm{BC}$, which was 
calculated based on the estimate of 10,420 HIV infected people in BC in HIV/AIDS Epi Updates (2007, Public Health Agency of Canada) and the population of 4,113,000 in BC. It is documented in the literature that TB contacts usually have a higher HIV infection rate than the general population. Thus this approach was also inadequate. The approach with the missing indicator included as an additional factor showed that the missingness of the HIV entry is significantly associated with time to TB. This confirmed the concern about the MCAR assumption. The associated analysis, however, provided only inferences on the effect of HIV status to time to TB among the subjects with available HIV entries. In summary, none of the initial analyses presented in Table 1 appeared satisfactory.

\subsection{Analysis with supplementary information}

Applying the methodology presented in the previous sections, we conducted analyses of the TB contact data using relevant HIV prevalence and demographic information in the literature. The first analysis assumed that overall HIV infection rate is independent of all the covariates under consideration. Thus $G(\cdot \mid z)=G(\cdot)$ is determined by $P(X=1)$, and the distribution of unobserved $X$ is determined by $P(X=1 \mid R=0)$. The study team used $1 \%$ as an estimate for HIV infection rate of TB contacts. We implemented the pseudo-likelihood approach described in Section 4 with the "true" overall HIV infection rate of TB contacts as $P(X=1)=0.01$. Moreover, in the analysis the proportion of

the subjects with available HIV status was $\hat{P}(R=1)=171 / 7754$ and the observed HIV infection rate was $\hat{P}(X=1 \mid R=1)=19 / 171$. This together with the estimated overall HIV rate of $1 \%$ gave $\tilde{P}(X=1 \mid R=0)=0.0077$. Table 2 presents the MPSLE estimates of the regression parameters in the Cox model, the estimates of the standard errors of the 
estimators obtained by the nonparametric bootstrap, and the p-values of the associated significance tests. In addition, we considered a realistic range of the HIV infection rate in all contacts of active TB cases in BC. The range was $0.3 \%$ (the HIV prevalence of the general population in Vancouver) to $9 \%$ (Reichler, et al, 2003, the highest HIV rate in TB contacts in the literature). To check the sensitivity of the analysis, we took the lower and the upper bound of the range as the true overall HIV rate, and implemented the pseudolikelihood approach accordingly. The associated analysis outcomes are presented in Table $2(\mathrm{a})$.

(Table 2 is here.)

The three sets of results are in agreement with respect to effects of all the risk factors except cluster-status of TB source cases. In particular, they reveal that HIV status, age at contact and level of contact are statistically significant risk factors but gender is not. As expected, the analysis indicates that HIV infected TB contacts are at a much higher risk of developing TB, and so are the TB contacts with high levels of contact. The analysis also suggests that older TB contacts are at a lower risk of TB than younger ones. An explanation for this finding is that older TB contacts were likely previously infected (exposed more than once especially if foreign-born), or infected many years ago so that they are well past the period of greatest risk for reactivation of TB (2-3 years post contact). This is consistent to what is well established in the literature that children are at greater risk for being infected once exposed, and progressing to active TB once infected, also to the more serious forms of TB. The factor of source case cluster-status, however, was found to be significantly associated with the time to TB when using the lower bound but not when using the estimate or the upper bound of the overall HIV infection rate. We should therefore be particularly 
cautious when interpreting the significance of the effect of the source case cluster-status.

Noting HIV infection rates are likely different across groups, we analyzed the TB contact data in two other scenarios, (i) $P(X=1 \mid z)=P(X=1 \mid$ gender) or (ii) $P(X=1 \mid z)=$ $P(X=1 \mid$ age $)$. Based on HIV/AIDS Epi Updates (2007, Public Health Agency of Canada), about $20 \%$ of HIV infected people are women, and the distribution of HIV infection across young $(0-24$ years old $)$, middle $(24-50$ years old), and old (50 or above) groups is $3.5 \%, 84.3 \%, 12.2 \%$. Using this information and the Canada's demographics information posted in the web site http://en.wikipedia.org/ yield the following.

(i) The ratio of $P(X=1 \mid$ gender $) / P(X=1)$ is 1.6 and 0.4 corresponding to the male and female groups, respectively.

(ii) The ratio of $P(X=1 \mid$ age $) / P(X=1)$ is $0.12,2.34$ and 0.35 corresponding to the young, middle and old age groups.

Combining the above with the estimates used for $P(X=1)$, the overall HIV infection rate in TB contacts, we implemented the procedure in Section 4.2. Table 2 (b) and (c) present the estimates and p-values in Scenario (i) and (ii), respectively. The two additional analyses confirm the significant associations of TB development with the three risk factors: HIV status, age at contact and contact level. In agreement with the first analysis, the assessments on the significance of the source type vary according to the overall HIV infection rate. Overall, the assessments of significance in Scenario (i) are close to the ones obtained in the analysis presented in Table 2 (a). We observe differences in the analysis in Scenario (ii). This third analysis shows that male TB contacts had significantly higher risks than females when the overall HIV infection rate is high (9\%). In addition, it reveals a stronger 
association of the source type with the TB development of TB contacts. This suggests a further investigation about how HIV status depends on other covariates, in particular, the age at the contact in the TB contact study.

\subsection{Additional sensitivity analysis}

It was of the primary interest in the $\mathrm{TB}$ contact study to evaluate the effect of source case cluster-status on the TB development of the contacts. The analysis presented above reveals that the effect significance depends on the assumed HIV infection rate of all study subjects. We conducted a sensitivity analysis to further explore the association. We sampled from the linearly transformed Beta-distribution, $2.7 \% \operatorname{Beta}(a, b)+0.3 \%$, with various combinations of the Beta parameters $(a, b)$ : $(1,30),(1,6),(1,3),(3,3),(3,1),(6,1),(30,1)$. All the samples ranged between $0.3 \%$ and $3 \%$ with the means associated with the above pairs of $(a, b)$ given by $0.39 \%, 0.68 \%, 0.97 \%, 1.7 \%, 2.3 \%, 2.6 \%, 2.9 \%$, respectively, since the $\operatorname{Beta}(a, b)$ distribution has mean $a /(a+b)$. With each of the realizations used as the "true" value of $P(X=1)$, the pseudo-likelihood approach in Section 4 was implemented to analyze the TB contact data under the three assumptions about $P(X=1 \mid Z)$ in Section 5.2. The resulting outcomes, in agreement with the results in Table 2 , were consistent with inferences on significance of all the covariates except for the cluster-status of TB source cases. The three plots in Figure 1 present the MPSLE estimates (points) along with the $2.5 \%$ and 97.5\% percentiles (the vertical line segments) for the regression coefficient of the clusterstatus of TB source cases. The results associated with each of the aforementioned pairs of $(a, b)$ were based on 30 repetitions and presented at the sample mean of the generated HIV rates. The plots show a trend of the dependence between estimated significance levels 
of the cluster-status factor and the assumed overall HIV infection rates in the analysis. In contrast to Figure 1 (a) and (b), Figure 1 (c) presents rather stable significance levels of the cluster-status factor with high HIV infection rates stratified according to the age at contact.

(Figure 1 is here.)

\section{Discussion}

When the missing covariate is missing at random (MAR), the approach in Section 3 reduces to the approach in Herring and Ibrahim (2001), which uses the EM algorithm and estimates the estimating function with the full covariate data. Specifically, in the E-step, Herring and Ibrahim use the approximation $\mathrm{E}\left[S_{1}(\theta) / S_{0}(\theta)\right] \approx \mathrm{E} S_{1}(\theta) / \mathrm{E} S_{0}(\theta)$, which leads to our estimating equations (9) and (10).

A variation of Algorithm 1 is to substitute Step 1B with the following:

Step 1B*: Evaluate $\widehat{x_{i}}$ in (11), and approximate $\widehat{e^{\alpha x_{i}}}$ and $\widehat{x_{i}} \widehat{e^{\alpha x_{i}}}$ by $e^{\widehat{x_{i}} \alpha}$ and $\widehat{x_{i}} e^{\widehat{x_{i} \alpha}}$, respectively.

This variation of ALGORITHM 1 can be viewed as applying iteratively the conventional partial likelihood approach under the Cox model with the missing $x_{i}$ imputed using the current estimates of $\alpha, \beta$ and $H_{0}(\cdot)$. It can be implemented in each iteration using the builtin functions in Splus or R for the Cox regression with fully observed covariates. Further investigation is required to study the asymptotic properties of the resulting estimators.

Practical applications of the procedure in Section 4 require that the dimension of $Z^{(1)}$ and the number of its distinct values are both small enough, compared to the sample size 
$n$, such that both $n_{1}\left(z^{(1)}\right)$ and $n\left(z^{(1)}\right)$ are reasonably large for each $z^{(1)}$. To choose an appropriate component vector of $Z$ as $Z^{(1)}$ when $X$ is an indicator, for example, we may consider the logistic regression model,

$$
P(X=1 \mid R=0, Z=z)=\exp \left(\eta^{\prime} z^{(*)}\right) /\left(1+\exp \left(\eta^{\prime} z^{(*)}\right)\right)
$$

with $z^{(*)}$ composited of 1 and a component vector of $z$. Extending Algorithm 1, we can then maximize the likelihood function (4), which now involves the parameter $\eta$, by solving the estimating equations (5) together with $\partial \log L\left(\alpha, \beta, h_{0}(\cdot) ; G_{0}\right) / \partial \eta=0$ to attain the MLE of $\alpha, \beta, h_{0}(\cdot)$ and $\eta$. The AIC and BIC variable selection procedures can be then applied based on (4). However, the implementation could be computationally intensive, especially when the dimension of $Z$ is large. An alternative approach is to extend to situations with missing covariates the method of Li, Dicker and Zhao (2010), who consider a new class of Dantzig selectors for linear regression models with censored responses.

This paper assumes that all of the individuals in the study are independent. However, in fact, the subjects in this example were clustered according to their TB source cases: TB contacts who had the same TB source case were likely correlated and grouped together. The current proposed approaches can be extended in principle to handle clustered study subjects, if a correlation structure is assumed. It is usually challenging to specify a plausible as well as feasible model to describe the correlation in practice.

The proposed approach requires the assumptions A1 and A2 in Section 2. There are practical situations where either of the two assumptions is violated. In such situations, the assumptions are probably plausible after having additional factors included in the covariate list. If the new covariates are observable, the proposed approach is then applicable with the 
new covariate list; otherwise, we may extend the proposed approach by applying a method for handling latent covariates in the literature.

As suggested by a referee, it would be worthwhile exploring how the resulting inference in Section 4 depends on the assumption of $G_{0}(\cdot \mid Z)=G_{0}\left(\cdot \mid Z^{(1)}\right)$, particularly when the true

model is $G_{0}(\cdot \mid Z)=G_{0}\left(\cdot \mid Z^{(2)}\right)$ with $Z^{(2)}$ a component vector of $Z$ and containing $Z^{(1)}$ as a component vector. Another further investigation is to develop Bayesian methodology for handling the covariates missing not at random (MNAR), which may naturally incorporate available prior information on the parameters.

\section{Acknowledgments}

The research was partially supported by grants from the Natural Sciences and Engineering Research Council of Canada (NSERC). We are very grateful to the editor, the associate editor and the two referees for their helpful comments and suggestions, which have greatly improved the paper. We thank Chunfang Lin for a preliminary numerical study and Jerry Lawless for a helpful discussion.

\section{References}

Andersen, P.K., Borgan, O., Gill, R.D. and Keiding, N. (1992). Statistical Models Based on Counting Processes, Springer, New York.

Bang, H. and Robins, J.M. (2005). "Doubly robust estimation in missing data and causal inference models," Biometrics 61, 962-973.

Breslow, N.E. (1972). "Discussion of the paper by D. R. Cox," J. R. Statist. Soc. B 34, 216-217.

Canadian Tuberculosis Standards 6th Edition. Edited by Long, R. Canadian Lung Association, Canadian Thoracic Society and Tuberculosis Prevention and Control Centre for Infectious Disease Prevention and Control, Health Canada; 2007. 
Chen, M.H., Ibrahim, J.G. and Shao, Q.M. (2009). "Maximum likelihood inference for the Cox regression model with applications to missing covariates," J. Multiv. Analys. 100, 2018-2030.

Chen, H.Y. and Little, R.J.A. (1999). "Proportional Hazards Regression with Missing Covariates," J. Amer. Statist. Assoc. 94, 896-908.

Cook, V.J., Hernandex-Garduno, E., Hu, X.J., Elwood, R.K. and FitzGerald, J.M. (2005). "The influence of cluster-status of source cases on contact evaluation and the development of secondary active tuberculosis," Proceedings of the American Thoracic Society (PATS) 2005; 2 Abstract Issue.

Cox, D.R. (1972). "Regression models and life tables (with Discussion)," J. R. Statist Soc. $B$ 34, 187-220.

Dempster, A.P., Laird, N.M. and Rubin, D.B. (1977). "Maximum likelihood from incomplete data via the EM algorithm," J. Roy. Statist. Soc. B 39, 1-22.

Efron, B. and Tibshirani, R.J. (1993). An Introduction to the Bootstrap, Monographs on Statistics and Applied Probability 57, CHAPMAN \& HALL/CRC.

Herring, A.H. and Ibrahim, J.G. (2001). "Likelihood-based methods for missing covariates in the Cox proportional hazards model," J. Amer. Statist. Assoc. 96, 292-302.

HIV/AIDS Epi Updates November 2007, Centre for Infectious Disease Prevention and Control, Public Health Agency of Canada.

Hu, X.J. and Lawless, J.F. (1996). "Estimation from truncated lifetime data with supplementary information on covariates and censoring times," Biometrika 83, 747-61.

Hu, X.J. and Lawless, J.F. (1997). "Pseudolikelihood estimation in a class of problems with response-related missing covariates," Can. J. Statist. 25, 125-42.

Li, Y., Dicker, L. and Zhao, S. (2010). "A new class of Dantzig selectors for censored linear regression models," Harvard University Biostatistics Working Paper Series (March 2, 2010).

Lin, D.Y. and Ying, Z. (1993). "Cox regression with incomplete covariate measurements," J. Amer. Statist. Assoc. 88, 1341-9.

Little, R.J.A. and Rubin, D.B. (2002). Statistical Analysis with Missing Data, Wiley Series in Probability and Statistics, Wiley.

Nan, B., Kalbfleisch, J.D. and Yu, M. (2009). "Asymptotic theory for the semiparametric accelerated failure time model with missing data," Ann. Statist. 37, 2351-2376.

Paik, M.C. and Tsai, W.Y. (1997). "On using the Cox proportional hazards model with missing covariates," Biometrika 84, 579-593.

Qi, L., Wang, C.Y., and Prentice, R.L. (2005). "Weighted estimators for proportional hazards regression with missing covariates," J. Amer. Statist. Assoc. 100, 1250-63. 
Reichler, M.R., Bur, S., Reves, R., Mangura, B., Thompson, V., Ford, J. and Castro, K.G. (2003). "Results of testing for human immunodeficiency virus infection among recent contacts of infectious tuberculosis cases in the United States," Int. J. Tuberc. Lung Dis. 7(12): 5471-5478.

Robins, J.M., Rotnitzky, A. and Zhao, L.P. (1994). "Estimation of regression coefficients when some regressors are not always observed," J. Amer. Statist. Assoc. 89, 846-66.

Scharfstein, D.O., Rotnitzky, A. and Robins, J.M. (1999). "Adjusting for nonignorable drop-out using semiparametric nonresponse models," J. Amer. Statist. Assoc. 94, 1096-1120.

Suzuki, K. (1985). "Estimation method of lifetime based on the record of failures during the warranty period," J. Amer. Statist. Assoc. 80, 68-72.

Tsiatis, A.A. (2006). Semiparametric Theory and Missing Data, Springer Series in Statistics, SPRINGER.

Zeng, D. and Cai, J. (2005). "Asymptotic results for maximum likelihood estimators in joint analysis of repeated measurements and survival time," Ann. Statist. 33, 21322163.

Zhou, H. and Pepe, M.S. (1995). "Auxiliary covariate data in failure time regression," Biometrika 82, 139-149. 
Table 1: Initial Analyses of the TB Contacts Study Data

\begin{tabular}{|c|c|c|c|c|c|c|c|c|}
\hline \multirow[t]{2}{*}{ Risk Factor } & \multicolumn{2}{|c|}{ HIV Status Removed } & \multicolumn{2}{|c|}{ Complete Cases Only } & \multicolumn{2}{|c|}{ Missing HIV Status Imputed } & \multicolumn{2}{|c|}{ Missing Indicator Introduced } \\
\hline & MPSLE & p-value & MPSLE & p-value & MPSLE & p-value & MPSLE & p-value \\
\hline Source Type (+ vs -) & 1.57 & $<0.01$ & 0.94 & 0.06 & 1.31 & $<0.01$ & 1.16 & $<0.01$ \\
\hline Gender (male vs female) & 0.19 & 0.46 & 0.29 & 0.51 & 0.14 & 0.58 & 0.12 & 0.65 \\
\hline HIV Status (+ vs -) & & & 0.74 & 0.25 & 3.57 & $<0.01$ & $1.45^{*}$ & $<0.01^{*}$ \\
\hline Age & -0.03 & $<0.01$ & -0.03 & 0.04 & -0.03 & $<0.01$ & -0.03 & $<0.01$ \\
\hline Contact Level & 1.20 & $<0.01$ & 0.23 & 0.44 & 1.24 & $<0.01$ & 1.02 & $<0.01$ \\
\hline Missing Indicator & & & . & & & & -2.48 & $<0.01$ \\
\hline
\end{tabular}

${ }^{*}$ Outcomes associated with HIV status within subjects with HIV entries. 
Table 2: Analyses of the TB Contacts Study Data with Assumed $G(\cdot \mid z)$

(a) Assuming $P(X=1 \mid Z=z)=P(X=1)$

\begin{tabular}{|c|c|c|c|c|c|c|c|c|c|}
\hline \multirow[t]{2}{*}{ Risk Factor } & \multicolumn{3}{|c|}{$P(X=1)=0.003$} & \multicolumn{3}{|c|}{$P(X=1)=0.01$} & \multicolumn{3}{|c|}{$P(X=1)=0.09$} \\
\hline & MPSLE & (s.e.) & p-value & MPSLE & (s.e.) & p-value & MPSLE & (s.e.) & p-value \\
\hline Source Type $(+$ vs -$)$ & 1.28 & $(0.29)$ & $<0.01$ & 0.36 & $(0.38)$ & 0.17 & 0.20 & $(0.34)$ & 0.28 \\
\hline Gender (male vs female) & 0.14 & $(0.26)$ & 0.29 & 0.09 & $(0.26)$ & 0.37 & 0.37 & $(0.27)$ & 0.09 \\
\hline HIV Status (+ vs -$)$ & 3.70 & $(0.47)$ & $<0.01$ & 5.94 & $(0.42)$ & $<0.01$ & 6.88 & $(0.36)$ & $<0.01$ \\
\hline Age at Contact & -0.03 & $(0.01)$ & $<0.01$ & -0.04 & $(0.01)$ & $<0.01$ & -0.05 & $(0.01)$ & $<0.01$ \\
\hline Contact Level & 1.25 & $(0.18)$ & $<0.01$ & 1.38 & $(0.18)$ & $<0.01$ & 1.24 & $(0.19)$ & $<0.01$ \\
\hline
\end{tabular}

(b) Assuming $P(X=1 \mid Z=z)=P(X=1 \mid$ gender $)$

\begin{tabular}{l|ccc|ccc|ccc}
\hline \multirow{2}{*}{ Risk Factor } & \multicolumn{3}{|c|}{$P(X=1)=0.003$} & \multicolumn{3}{c|}{$P(X=1)=0.01$} & \multicolumn{3}{c}{$P(X=1)=0.09$} \\
\cline { 2 - 11 } & MPSLE & $($ s.e. $)$ & p-value & MPSLE & (s.e.) & p-value & MPSLE & $($ s.e. $)$ & p-value \\
\hline Source Type (+ vs -) & 1.29 & $(0.29)$ & $<0.01$ & 0.47 & $(0.37)$ & 0.10 & 0.18 & $(0.34)$ & 0.29 \\
Gender (male vs female) & 0.14 & $(0.26)$ & 0.30 & 0.06 & $(0.26)$ & 0.42 & -0.25 & $(0.27)$ & 0.83 \\
HIV Status (+ vs -) & 3.66 & $(0.47)$ & $<0.01$ & 5.73 & $(0.42)$ & $<0.01$ & 6.86 & $(0.35)$ & $<0.01$ \\
Age at Contact & -0.03 & $(0.01)$ & $<0.01$ & -0.04 & $(0.01)$ & $<0.01$ & -0.05 & $(0.01)$ & $<0.01$ \\
Contact Level & 1.25 & $(0.18)$ & $<0.01$ & 1.36 & $(0.18)$ & $<0.01$ & 1.40 & $(0.19)$ & $<0.01$ \\
\hline
\end{tabular}

(c) Assuming $P(X=1 \mid Z=z)=P(X=1 \mid$ age $)$

\begin{tabular}{l|ccc|ccc|ccc}
\hline \hline \multirow{2}{*}{ Risk Factor } & \multicolumn{3}{|c|}{$P(X=1)=0.003$} & \multicolumn{3}{c|}{$P(X=1)=0.01$} & \multicolumn{3}{c}{$P(X=1)=0.09$} \\
\cline { 2 - 10 } & MPSLE & $($ s.e. $)$ & p-value & MPSLE & $($ s.e. $)$ & p-value & MPSLE & $($ s.e. $)$ & p-value \\
\hline Source Type (+ vs -) & 1.27 & $(0.29)$ & $<0.01$ & 0.61 & $(0.36)$ & 0.04 & 0.31 & $(0.35)$ & 0.19 \\
Gender (male vs female) & 0.14 & $(0.26)$ & 0.30 & 0.15 & $(0.26)$ & 0.28 & 1.05 & $(0.30)$ & $<0.01$ \\
HIV Status (+ vs -) & 3.75 & $(0.46)$ & $<0.01$ & 5.54 & $(0.41)$ & $<0.01$ & 6.64 & $(0.37)$ & $<0.01$ \\
Age at Contact & -0.03 & $(0.01)$ & $<0.01$ & -0.04 & $(0.01)$ & $<0.01$ & -0.05 & $(0.01)$ & $<0.01$ \\
Contact Level & 1.25 & $(0.18)$ & $<0.01$ & 1.35 & $(0.18)$ & $<0.01$ & 1.35 & $(0.19)$ & $<0.01$ \\
\hline
\end{tabular}



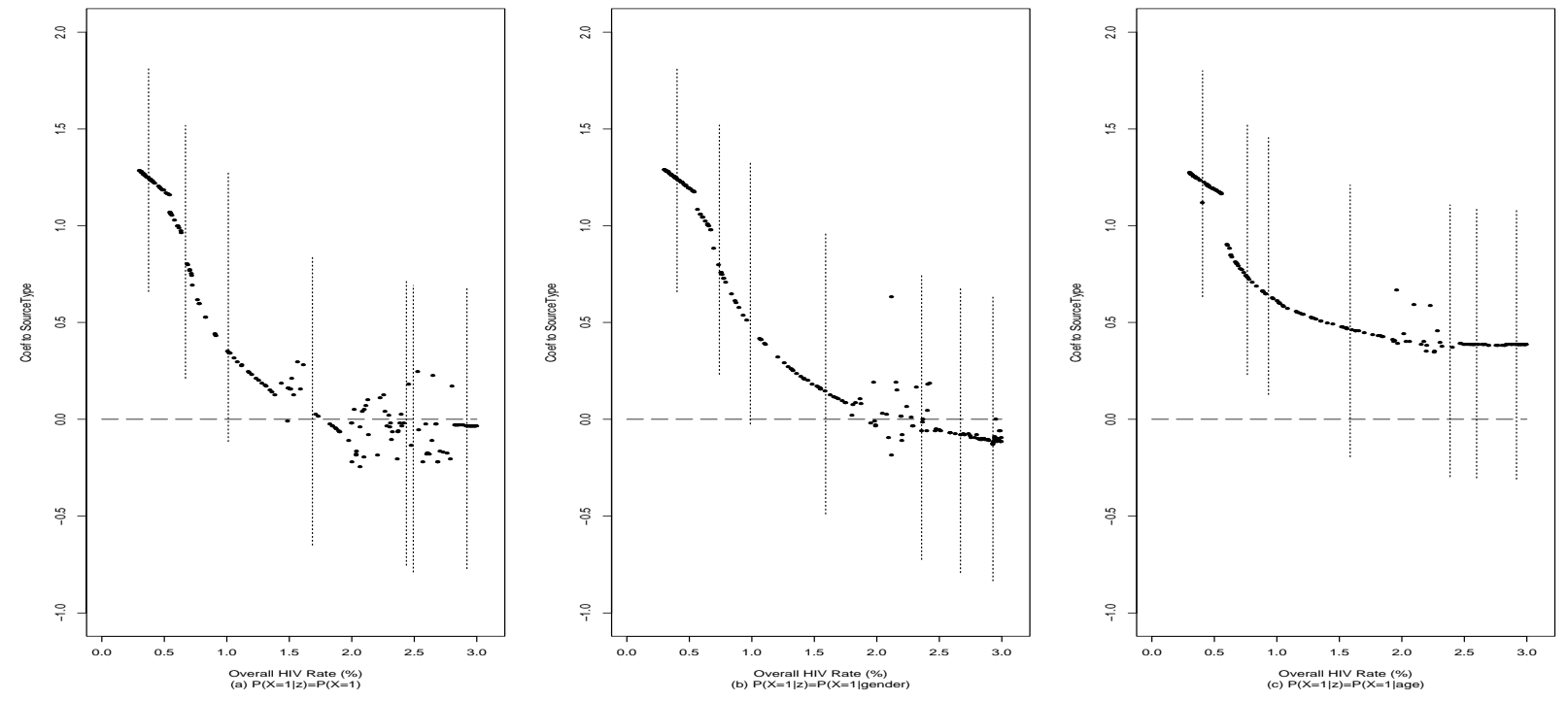

Figure 1: Sensitivity Analysis: MPSLE estimates for the coefficient of cluster-status of TB source cases with varying overall HIV + rates in the TB contacts study 